\title{
Post-stroke disposition from a geriatric-rehabilitative stroke care area: an Italian experience
}

\author{
Marco Masina, ${ }^{1}$ Simona Malservisi, ${ }^{1}$ Pio Francesco Censoni, ${ }^{2}$ Annalena Cicognani, ${ }^{1}$ Paolo Marzaroli, ${ }^{3}$ \\ Paolo Pandolfi, ${ }^{3}$ Francesca Ugenti ${ }^{4}$
}

${ }^{1}$ UOC Geriatria Bentivoglio, Azienda USL di Bologna; ${ }^{2}$ UOC Medicina Riabilitativa Nord, Azienda USL di Bologna; ${ }^{3}$ UOC Epidemiologia, Promozione della Salute e Comunicazione del Rischio, Azienda USL di Bologna; ${ }^{4}$ UOS Lungodegenza Nord, Azienda USL di Bologna, Italy

\begin{abstract}
A large number of stroke patients cannot be discharged at home. Studies on post stroke disposition have low validity outside the country in which they are carried out because healthcare systems offer different rehabilitative and long-term facilities. Moreover absolute selection criteria for admission to rehabilitation are not available yet. Few studies on post-stroke disposition from Italian stroke units are available. Authors evaluated data of a 18-month period from a geriatric managed stroke care area where comprehensive multi-professional assessment and discharge planning are routinely carried out. Only patients discharged with diagnosis related to acute stroke were considered. Baseline characteristics, clinical, neurological and functional conditions according to the structured multidimensional assessment were prospectively collected in the stroke unit registry. Univariate and multinomial logistic regression were performed to identify independent variables associated with three discharge settings: home, rehabilitation and skilled long-term ward. Out of 188 patients evaluated, $56.4 \%$ were discharged home, $18.6 \%$ to rehabilitation and $25.0 \%$ to long-term ward. Data showed an efficient disposition to intermediate settings with a shorter length of stay compared to other international studies. Factors associated with post-stroke disposition were age, dysphagia, neurological impairment on admission (NIH-SS $\geq 6$ ), after stroke functional status (mRankin $\geq 3$ ), poor pre-stroke functional level (mRankin $\geq 3$ ) and hemorrhagic stroke. Dysphagia, severe neurological impairment and post-stroke disability were associated with discharge to rehabilitation and long term ward. These two settings differed in age and pre-stroke functional condition. Patients discharged to long-term wards were about 10 years older than those admitted to rehabilitative ward. Only $5 \%$ of patients discharged to rehabilitation had a pre-stroke mRankin score $\geq 3$. Disposition to a skilled longterm ward of older patients with pre-stroke disability seemed positive on an economic ground but further studies are mandatory to understand the consequences in terms of functional recovery and social costs.
\end{abstract}

\section{Introduction}

Stroke is a main challenge for health care systems because its prevalence increases in older people. ${ }^{1}$ Aiming at reducing mortality and disability many countries have developed a network system of stroke units dedicated to the acute management of stroke patients. ${ }^{2,3}$ In stroke unit a specialized multi-professional team focuses on direct stroke treatment (systemic fibrinolysis, endovascular revascularization, vascular surgery) and ensures quick team activation, specialized nursing care, effective management of dysphagia, prevention of complications, and early rehabilitation. ${ }^{2}$ 
Nevertheless patients develop functional deficits and dependence in a percentage ranging from 15 to $30 \%$ and cannot return home because they need individual rehabilitative treatment and/or management of disability. ${ }^{1}$ Post-stroke disposition affects the length of stay (LOS) and the appropriate allocation of resources.

International literature reports several studies on predictive criteria of post-stroke disposition. In the USA, Israel and Scandinavia three main discharge settings (home, rehabilitation department and nursing home) are considered. ${ }^{4-9}$ In France authors differentiate among four settings: home, rehabilitation, convalescent (temporary) home and long-term nursing home. ${ }^{10}$ Main factors associated with post-stroke disposition are the severity of neurological deficit at the entrance, ${ }^{4,7,8}$ age, LOS in stroke units, functional impairment of the upper arm and/or aphasia, ${ }^{9}$ the level of disability at discharge, ${ }^{11}$ incontinence, the need of feeding probe and cognitive impairment. ${ }^{12}$ In a recent systematic review Hakkennes highlighted the most important indicators of disposition from a stroke unit [age, cognitive status, National Institute of Health Stroke Scale (NIH-SS) score, after stroke functional status and comorbidity] and the indicators of admission to rehabilitation (after stroke impairment, prestroke functional level and cognitive status). ${ }^{13}$ Nevertheless absolute selection criteria for rehabilitation admission remain not available. ${ }^{14}$

In 2010, according to the Emilia Romagna regional stroke care program, a new stroke area opened in the Bentivoglio Hospital, which offers in-patient cares to the 150,000 inhabitants in the northern suburban province of Bologna. The stroke area was implemented inside the acute geriatric ward, which insisted in a geriatric-rehabilitative area that encompassed also two post-acute long-term wards and a rehabilitation ward. In our region, post-acute long-term wards are in-hospital settings that offer temporary skilled nursing care and low intensive physiotherapy to patients with disability not eligible to individual rehabilitative treatment. In post-acute wards a discharge planning is carried out in order to foster the patient's return home. The geriatric ward of the Bentivoglio Hospital has a undisputed competence in multi-professional team working and in multidimensional assessment. ${ }^{15}$ Geriatricians and geriatric nurse staff grant comprehensive care and direct management through the multidisciplinary approach of comprehensive geriatrics assessment. Since the opening of the stroke care area, a clinical governance program has been implemented and an audit continuously monitors patients' care, treatments and disposition. ${ }^{15}$

Few studies on post-stroke disposition are available from Italian stroke units. ${ }^{16}$ The most recent ones only evaluated predictors to clinical outcomes or to disposition to rehabilitative treatment excluding the discharge to long-term ward. ${ }^{17,18}$ In the present study the authors evaluated a stroke unit in which comprehensive geriatric and multi-professional assessment and discharge planning are carried out. Baseline, clinical and post-stroke factors and length of stay were analyzed in order to provide a complete representation of how they correlated to the discharge settings.

\section{Materials and Methods}

Authors evaluated the activity of the stroke care area of the Bentivoglio Hospital, Italy from January 1, 2011 to June 30, 2012. All patients admitted to the stroke unit with documented brain injury from ischemic stroke or intracerebral hemorrhage and discharged with diagnosis related to acute cerebrovascular accident were considered. Patients with transient ischemic attack, subarachnoid hemorrhage and cerebral venous sinus thrombosis were excluded.

Subjects were excluded if they deceased in hospital, and if they were hosted in nursing home or were totally dependent (modified Rankin score $=5$ ) before the index event. Patients were also excluded if previously admitted to other stroke units or whether disposing data were missing. Due to their small number, patients that received thrombolytic therapy were excluded too.

All patients underwent a structured multidimensional assessment. Characteristics of patients and indicators of comprehensive assessment were prospectively collected in a registry. A systematic internal audit continuously monitored indicators of quality. The results largely fulfilled the National Institute of Clinical Excellence (NICE) Quality Standard bundles for stroke and were reported in a previous study. ${ }^{15}$ A team with a geriatrician, a nurse, a physiatrist and a physiotherapist visits stroke patients within $36 \mathrm{~h}$ from admission and patients start rehabilitation as soon as clinical conditions allow it. The multi-professional approach continues also in post-acute long-term ward where both nursing care and a low intensive physiotherapy are granted to patients with disabilities that cannot sustain more intensive rehabilitative programs.

Identification of patients and collection of data and indicators were based on the ward registry. For missing data, medical records of patients were reviewed. Patients' discharge pathway was assessed by the hospital log. Three settings of discharge were considered: home, rehabilitative ward and post-acute long-term ward. The study was approved by the Local Ethical Committee.

\section{Statistical analysis}

Univariate analyses were performed to test the relationship among variables and each setting of discharge. Continuous variables have been tested using 
the analysis of variance (ANOVA) and those categorical using the $\chi^{2}$ test or the Fischer test when indicated. All tests were two-tailed. Variables significant at univariate analysis $(\mathrm{P}<0.05)$ were considered for the multivariate analysis. Multinomial analyses were performed to identify independent variables associated with each setting of discharge for comparison to the discharge at home. All analyses were performed using STATA version 12.

\section{Results}

During the 18-month period, 260 patients were admitted to the stroke area. Among them 9 had stroke mimic, 31 had transient ischemic attack, 1 had subarachnoid hemorrhage, 2 lived in nursing home before stroke, 2 received intravenous thrombolysis, 20 expired in stroke care area and 7 were urgently referred to vascular surgeon or cardiologist.

Of the 188 remaining patients, 106 (56.4\%) were discharged home, $35(18.6 \%)$ to rehabilitation ward and $47(25.0 \%)$ to long-term wards. Their characteristics are described in Table 1.

The mean age was $77.9 \pm 10.9$; male were 101 $(53.7 \%)$. Social condition before stroke: $51.6 \%$ lived alone; $28.2 \%$ lived with spouse and $20.2 \%$ lived with relatives or had in-home care. The average NIH-SS score on admission was $6.78 \pm 6.93$ (range $\min 0$ - $\max 42$ ).

Patients referred to post-acute long-term ward were significantly older $(84.8 \pm 5.9)$ than those discharged home $(75.9 \pm 11.4)$ or to rehabilitation ward (74.7 \pm 10.6$)$. Patients were significantly discharged to long-term wards if they were female ( $\mathrm{P}=0.02)$, had a pre-stroke diagnosis of dementia $(\mathrm{P}<0.001)$ or a worse pre-stroke disability condition as assessed by anamnestic modified Rankin score $\geq 3(\mathrm{P}<0.001)$. Living with relatives and having in-home care before stroke showed no significance to post-stroke disposition $(\mathrm{P}=0.06)$. Anamnestic prior strokes $(\mathrm{P}=0.68)$ and Charlston comorbidity index with a categoriza-

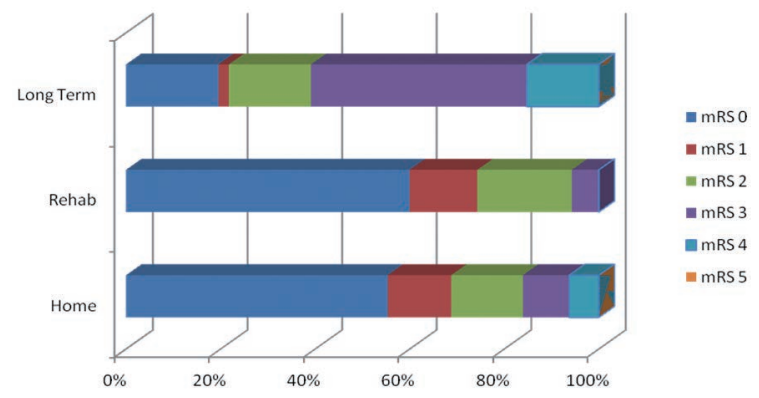

Figure 1. Distribution of pre-stroke mRankin score (mRS) (patients with pre-stroke $\mathrm{mRS}=5$ not admitted to stroke unit). tion of $0-1$ and $\geq 2$ showed no significant correlation.

According to literature, ${ }^{4-7,9-13,19}$ the NIH-SS score on admission highly correlated to hospital disposition $(\mathrm{P}<0.0001)$. Among patients discharged home 76\% had a NIH-SS score $\leq 5,22 \%$ had NIH-SS score $\geq 6$ 13 and only $2 \%$ had a score $>13$. Among patients admitted to rehabilitation $46 \%$ had NIH-SS score $\leq 5$, $34 \%$ had a NIH-SS score $\geq 6-13$ and $20 \%$ had a NIH$\mathrm{SS}>13$. Of those discharged to long-term ward $26 \%$ had NIH-SS score $\leq 5,46 \%$ had a NIH-SS score $\geq 6$ 13 and $28 \%$ had a NIH-SS $>13$. Dysphagia $(\mathrm{P}<0.0001)$ but not aphasia was associated with discharge to intermediate care settings. ${ }^{20}$

Cardioembolic stroke patients were significantly discharged to long-term ward $(\mathrm{P}=0.02)$, lacunar stroke were significantly disposed home $(\mathrm{P}=0.03)$; patients with total anterior circulation infarct were significantly discharged to intermediate care settings $(\mathrm{P}<0.001)$. Inhospital complications were significantly more frequent in patients who were not discharged home $(\mathrm{P}<0.001)$.

LOS in stroke unit was shorter in patients discharged home (8.6 63.1 days) than in patients sent to rehabilitation (12.4 \pm 6.5 days) or to long-term wards (10.4 \pm 4.6 days). Univariate analysis showed significant difference only between home and rehabilitation $(\mathrm{P}<0.001)$.

Functional status at discharge from stroke unit according to mRankin score was significantly better in patients who went home $(\mathrm{P}<0.001)$. More than $75 \%$ of those admitted to rehabilitation had disability in activity of daily living or were unable to walk alone (mRankin score $\geq 3$ ). The percentage raised to $93 \%$ in those discharged to long-term ward. Bars in Figures 1 and 2 show the distributions of mRankin score on admission and at discharge.

The overall length of stay in hospital ranged from $9.1 \pm 3.6$ for those discharged home, to $30.5 \pm 17.9$ for those discharged to post-acute long-term ward, and to $43.5 \pm 22.9$ for those admitted to rehabilitation $(\mathrm{P}<0.0001)$.

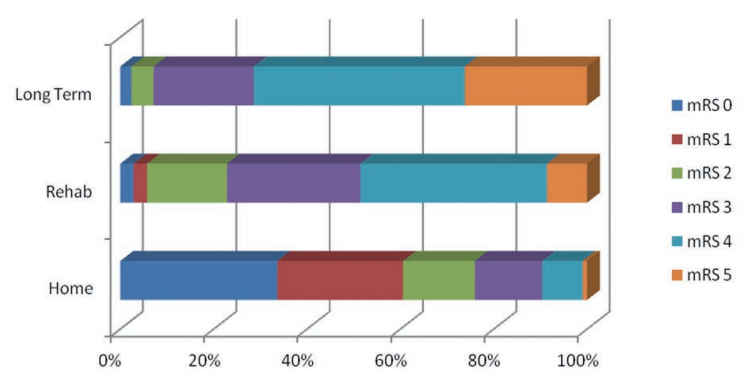

Figure 2. Distribution of post-stroke mRankin score (mRS). 
Table 1. Characteristic of patients according to disposition from stroke unit (univariate analysis).

\begin{tabular}{|c|c|c|c|c|}
\hline Baseline characteristics & Home & Rehabilitation & Long-term ward & $\mathbf{P}$ \\
\hline N. of patients: total $188(\%)$ & $106(56.4 \%)$ & $35(18.6 \%)$ & $47(25.0 \%)$ & \\
\hline Age (y) & $75.9 \pm 11.4$ & $74.7 \pm 10.6$ & & 0.82 \\
\hline Age (y) & $75.9 \pm 11.4$ & & $84.8 \pm 5.9$ & $<0.001$ \\
\hline Age (y) & & $74.7 \pm 10.6$ & $84.8 \pm 5.9$ & $<0.001$ \\
\hline Female sex & 37.7 & 51.4 & 61.7 & 0.02 \\
\hline Social status & & & & 0.06 \\
\hline Living alone & 17.9 & 22.9 & 23.4 & \\
\hline With spouse & 63.9 & 54.3 & 34.0 & \\
\hline Other relatives/home care & 23.6 & 22.9 & 42.6 & \\
\hline Dementia before stroke & 7.6 & 2.9 & 33.3 & $<0.001$ \\
\hline Comorbidity & & & & 0.745 \\
\hline $\mathrm{CCI}=0$ & 66.0 & 77.1 & 63.8 & \\
\hline $\mathrm{CCI}=1$ & 10.4 & 5.7 & 10.6 & \\
\hline $\mathrm{CCI} \geq 2$ & 23.6 & 17.1 & 25.5 & \\
\hline Prior stroke & 13.7 & 8.8 & 15.2 & 0.688 \\
\hline Disability prior to stroke & & & & $<0.001$ \\
\hline mRankin score before stroke $0-2$ & 84.9 & 97.1 & 46.8 & \\
\hline mRankin score before stroke 3-5 & 15.1 & 2.9 & 53.2 & \\
\hline
\end{tabular}

Admission stroke factors

Acute neurological evaluation (NIH-SS score)

NIH-SS $\leq 5$

NIH-SS 6-13

NIH-SS $>13$

Neurologic symptoms

Aphasia

Dysphagia

Type of stroke (TOAST)

Intracerebral hemorrhage

Cardioembolism

Lacunar

Large-artery atherosclerosis

Indeterminate

Other

OCSP classification

Total anterior cerebral infarction

In-hospital complications

Length of stay in stroke unit (days)

Length of stay in stroke unit

Length of stay in stroke unit

Length of stay in stroke unit

\section{Post-stroke factors}

mRankin score at disposition

mRankin score 0-2

mRankin score 3-5

\section{Overall in-hospital length of stay}

Mean \pm SD

$9.1 \pm 3.6$
$9.1 \pm 3.6$

79.1

20.9

$8.6 \pm 3.1$

$8.6 \pm 3.1$

76.4

21.7

45.7

34.3

20.0

19.0

13.2

25.7

45.7

47.8

0.112

$<0.001$

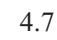

19.8

18.9

14.1

36.8

5.7

1.9

8.6

22.8

8.6

5.7

48.6

5.7

10.6

0.055

0.376

0.024

0.032

0.380

0.218

0.248

21.3

$<0.001$

53.2

$<0.001$

0.07

0.10

$12.4 \pm 6.5$

$10.4 \pm 4.6$

$<0.001$

7.0

93.0

$\begin{array}{cc}28.6 & 7.0 \\ 71.4 & 93.0\end{array}$

$43.5 \pm 22.9$

$30.5 \pm 17.9$

$<0.001$

$30.5 \pm 17.9$

$<0.001$

$43.5 \pm 22.9$

$<0.001$

CCI, Charlson comorbidity index; mRankin score, modified Rankin score; NIH-SS, National Institute of Health Stroke score; TOAST, Trial of Org10172 in Acute Stroke Treatment; OSCP, Oxfordshire Community Stroke Project classification; SD, standard deviation. Values are percent or mean \pm SD. 


\section{Multivariate analysis}

Two multivariate multinomial models analyzed factors associated with home disposition versus discharge to rehabilitative ward or to post-acute longterm ward.

The first model considered demographic variables (age, sex, social status), baseline and stroke related indicators on admission that were significant at univariate analysis: pre-stroke anamnestic mRankin, pre-stroke diagnosis of dementia, NIH-SS score on admission, stroke type, dysphagia (Table 2). Oxforshire Community Stroke Project classification (OCSP) types were excluded from analysis because the NIHSS score has a strong concurrent validity with respect to infarct volume that might influence results. ${ }^{21,22} \mathrm{Hav}-$ ing home disposition as referent, factors associated with rehabilitation were dysphagia [odds ratio (OR) 4.5 (95\% confidence interval, CI 1.68-12.13) $\mathrm{P}=0.003$ ], NIH-SS score $\geq 6-13$ [OR $2.91 \quad(95 \%$ CI $1.05-8.04) \mathrm{P}=0.04$ ], NIH-SS score $>13$ [OR $23.5(95 \%$ CI 4.11-134.83) $\mathrm{P}>0.001]$, pre-stroke mRanking score $<3$ [OR $0.10(95 \%$ CI $0.01-0.80) \mathrm{P}=0.03$ ]. Factors associated with discharge to long-term ward were dysphagia [OR $4.31(95 \%$ CI $1.35-13.72) \mathrm{P}=0.013$ ], NIH-SS score $\geq 6-13$ [OR 3.47 (95\% CI 1.16-10.41) $\mathrm{P}=0.03$ ], intra cerebral hemorrhage [OR $4.14(95 \% \mathrm{CI}$ $1.10-15.64) \mathrm{P}=0.04$ ] and age [OR 1.09 (95\% CI 1.001.19) $\mathrm{P}=0.04]$.

A second model added three stroke dependent covariates represented by in-hospital complications, post- stroke disability and LOS in stroke unit (Table 3). In this model, factors associated with rehabilitation were post-stroke mRankin score $\geq 3$ [OR $46.82(95 \%$ CI $10.03-218.60$ ] $\mathrm{P}<0.0001)$, dysphagia [OR 5.34 (95\% CI 1.48-19.31) $\mathrm{P}<0.01)$, younger age [OR $0.93(95 \% \mathrm{CI}$ 0.89-0.98, $\mathrm{P}<0.009$ ) and pre-stroke mRankin $<3$ [OR 0.03 (95\% CI $0.00-0.28, \mathrm{P}<0.002]$. Factor associated with discharge in long-term ward were post-stroke mRankin score $\geq 3$ [OR 68.61 (95\% CI 13.01-361.75) $\mathrm{P}<0.0001$ ] and dysphagia [OR 3.64 (95\% CI 1.0013.31) $\mathrm{P}<0.05$ ].

The results of the two models were coherent: dysphagia, neurological impairment, poor post-stroke functional status were associated with disposition to intermediate care settings. Younger age and good prestroke functional status were related to rehabilitation. Older age and hemorrhagic stroke were associated with discharge to long-term wards.

\section{Discussion}

According to literature a proportion of patients ranging from $25 \%$ to $45 \%$ cannot be discharged home after an acute stroke and are admitted to rehabilitation or to temporary/long term facilities or nursing home..$^{5,8,10}$ The characteristics of post-stroke settings vary from country to country and sometimes within the same country accounting for the differences reported in epidemiological studies. Those differences may significantly affect the LOS., $4,5,10$

Table 2. Multinomial regression analysis of admission characteristics associated with post-stroke disposition (patients No. $\left.179-R^{2}=0.2938\right)$.

\begin{tabular}{|c|c|c|c|c|c|c|c|c|}
\hline Variables & Notes & $\begin{array}{c}\text { Home } \\
\text { Reference }\end{array}$ & OR & $\begin{array}{c}\text { Rehabilitation } \\
(95 \% \text { CI })\end{array}$ & $\mathbf{P}$ & OR & $\begin{array}{c}\text { Long-term ward } \\
(95 \% \mathrm{CI})\end{array}$ & $\mathbf{P}$ \\
\hline Age & & 1 & 0.06 & $(0.93-1.00)$ & 0.13 & 1.09 & $(1.00-1.19)$ & $0.04 * *$ \\
\hline Male & Female as reference & 1 & 0.55 & $(0.22-1.41)$ & 0.21 & 1.07 & $(0.30-3.84)$ & 0.92 \\
\hline Spouse & Alone as reference & 1 & 1.46 & $(0.39-5.47)$ & 0.57 & 0.67 & $(0.19-2.40)$ & 0.55 \\
\hline Relat/home care & Alone as reference & 1 & 1.33 & $(0.42-4.21)$ & 0.63 & 0.72 & $(0.17-3.04)$ & 0.91 \\
\hline Pre-stroke dementia & No dementia as reference & 1 & 0.44 & $(0.02-9.49)$ & 0.60 & 2.00 & $(0.50-8.01)$ & 0.33 \\
\hline Prior stroke & No prior stroke as reference & 1 & 0.99 & $(0.22-4.49)$ & 0.99 & 0.69 & $(0.16-3.04)$ & 0.62 \\
\hline Pre-stroke mRankin 3-4 & mRankin $0-2$ referent & 1 & 0.10 & $(0.01-0.80)$ & $0.03 *$ & 2.93 & $(0.84-10.30)$ & 0.09 \\
\hline $\mathrm{ICH}$ & $\mathrm{ICH} v s$ ischemic & 1 & 2.42 & $(0.58-10.06)$ & 0.22 & 4.14 & $(1.10-15.64)$ & $0.04 * *$ \\
\hline Cardioembolism & $v s$ other TOAST types & 1 & 1.14 & $(0.36-3.54)$ & 0.82 & 2.56 & $(0.85-7.69)$ & 0.09 \\
\hline NIH-SS score $\geq 6-13$ & $\mathrm{NIH}-\mathrm{SS} \leq 5$ referent & 1 & 2.91 & $(1.05-8.04)$ & $0.04 *$ & 3.47 & $(1.16-10.41)$ & $0.03 * *$ \\
\hline NIH-SS score $>13$ & $\mathrm{NIH}-\mathrm{SS} \leq 5$ referent & 1 & 23.5 & $(4.11-134.83)$ & $<0.001 *$ & 8.16 & $(0.92-72.41)$ & 0.06 \\
\hline Dysphagia & No dysphagia & 1 & 4.51 & $(1.68-12.13)$ & $0.003 *$ & 4.31 & $(1.35-13.72)$ & $0.01 * *$ \\
\hline
\end{tabular}

OR, odds ratio; CI, confidence interval; mRankin, modified Rankin score; ICH, intracerebral hemorrhage; TOAST, Trial of Org10172 in Acute Stroke Treatment classification; NIHSS, National Institute of Health Stroke score. *Significant to rehabilitation ward: pre-stroke mRankin 3-4, NIHSS score >5, dysphagia; **Significant to long-term ward: age, ICH, NIH-SS score $\geq 6-13$, dysphagia. 
Patients discharged to rehabilitation or nursing home usually stay in stroke unit longer than those disposed home. ${ }^{5,10}$ In a recent population-based study in Dijon, France $(152,000$ inhabitants) a longer LOS was independently associated with discharge to rehabilitative ward and to convalescent home. ${ }^{10}$ In the Israel National Survey the average LOS in primary hospital was of $8.7 \pm 13.5$ days but it raised to 10 days in those admitted to rehabilitation and almost doubled in those discharged to nursing home. ${ }^{5}$ The delays were attributed to clinical conditions (severity of neurological damage, longer time to medical stabilization) but also to the accessibility to beds in rehabilitation or nursing home..$^{5,10}$

Our geriatric-rehabilitative area encompasses a stroke unit, 14 beds of rehabilitative ward and 40 beds in two long-term wards. After the acute stroke about $44 \%$ of patients were disposed other than home; $18.6 \%$ went to rehabilitation and $25.0 \%$ to long-term wards. Our organization seems to support an efficient disposition to intermediate settings. The LOS in our stroke unit was shorter and in multinomial analysis did not correlates to the discharge setting.

In our analysis the main factors associated with post-stroke disposition were age, dysphagia, severe neurological impairment on admission, severe after stroke functional status, poor pre-stroke functional level and hemorrhagic stroke. The association of dysphagia with a long in-hospital stay is consistent with literature, being dysphagia a dangerous condition that needs competent nursing (specific diets, tube feeding, etc.) and physiotherapeutic treatments to reduce complications and morbidity. Severe neurological status and poor post-stroke functional conditions are well established factors of disposition to intermediate care, but often authors complain that the most severe conditions are sent to convalescent home or nursing home and not to rehabilitation. ${ }^{10}$ That was not the case. In our experience both conditions were strongly associated with discharge to rehabilitation (Tables 2 and 3). The main differences between rehabilitative wards and long-term wards were found in age, hemorrhagic stroke and pre-stroke functional condition. Patients disposed to long-term wards were about 10 years older than those admitted to rehabilitative ward. Only 5\% of patients discharged to rehabilitation had a prestroke mRankin score $\geq 3$. The role of age is frequently reported in literature ${ }^{23-26}$ but it was quite unexpected in a geriatric managed stroke care area. A possible explanation may depend on organizational factors. Despite the multi-professional team, only the physiatrist can plan a rehabilitative program. The limited number of rehabilitative beds, the longer waiting time and the availability of a low intensive physiotherapy program in long-term wards might have lead physiatrists to select patients with better pre-stroke conditions and younger age in whom a valid functional recovery was more likely. ${ }^{10,13}$ That choice had important economic effects. The overall in-hospital LOS was of $30.5 \pm 17.9$ days for patients discharged to long-term ward and of $43.5 \pm 22.9$ days for those admitted to rehabilitation ward. The daily reimbursement for in-hospital rehabilitation in Italy near doubles the reimbursement for long-term ward accounting for a difference of about $€$ $10,000 /$ patient. The effect of the disposition to a skilled long-term ward of older patients with prestroke disability seems positive on an economic ground but further studies are warranted to better understand the consequences in terms of functional recovery and social costs.

Table 3. Multinomial regression analysis of characteristics at discharge associated with post-stroke disposition (patients No. $\left.176-R^{2}=0.4295\right)$.

\begin{tabular}{lcccccccc}
\hline Variables & Notes & $\begin{array}{c}\text { Home } \\
\text { Reference }\end{array}$ & OR & $\begin{array}{c}\text { Rehabilitation } \\
(\mathbf{9 5 \%} \text { CI) }\end{array}$ & P & OR & $\begin{array}{c}\text { Long-term ward } \\
\mathbf{( 9 5 \%} \text { CI) }\end{array}$ & P \\
\hline Age & & & 0.93 & $(0.89-0.98)$ & $0.009^{*}$ & 1.03 & $(0.94-1.14)$ & 0.50 \\
\hline Male & Female as reference & 1 & 0.78 & $(0.22-2.78)$ & 0.70 & 0.81 & $(0.22-2.93)$ & 0.74 \\
\hline Spouse & Alone as reference & 1 & 1.04 & $(0.19-5.61)$ & 0.96 & 0.52 & $(0.13-2.05)$ & 0.35 \\
\hline Relat/home care & Alone as reference & 1 & 1.55 & $(0.31-7.87)$ & 0.59 & 1.13 & $(0.24-5.25)$ & 0.87 \\
\hline Dementia & No dementia as reference & 1 & 0.31 & $(0.03-3.25)$ & 0.33 & 2.04 & $(0.51-8.21)$ & 0.31 \\
\hline Pre-stroke mRankin 3-4 & mRankin 0-2 referent & 1 & 0.03 & $(0.00-0.28)$ & $0.002^{*}$ & 0.64 & $(0.17-2.46)$ & 0.51 \\
\hline Complications & No complication & 1 & 1.31 & $(0.39-4.43)$ & 0.66 & 2.33 & $(0.75-7.27)$ & 0.14 \\
\hline Dysphagia & No dysphagia & 1 & 5.34 & $(1.48-19.31)$ & $0.01^{*}$ & 3.64 & $(1.0-13.31)$ & $0.05^{* *}$ \\
\hline mRankin 3-4 discharge & mRankin 0-2 referent & 1 & 46.82 & $(10.03-218.60)$ & $<0.000^{*}$ & 68.61 & $(13.01-361.75)$ & $<0.000^{* *}$ \\
\hline LOS in Stroke Unit & & & 1.07 & $(0.95-1.20)$ & 0.22 & 0.95 & $(0.83-1.08)$ & 0.40
\end{tabular}

OR, odds ratio; CI, confidence interval; mRankin, modified Rankin score; LOS, length of stay. *Significant to rehabilitation ward: age, pre-stroke mRankin 0-2, dysphagia, mRankin $\geq 3$ at discharge; $* *$ Significant to long-term ward: dysphagia, mRankin $\geq 3$ at discharge. 


\section{Study limitation}

In our study, a single stroke unit located in a geriatric-rehabilitative area was considered, so results can be assumed as indicative of a regional condition and do not represent the whole Italian situation. Another limitation may depend on the quality of our long-term wards. The low intensive physiotherapy performed in our long-term ward may have influenced the choice of the physiatrist. A larger number of patients might have allowed a better understanding of the role of other factors like cognitive impairment in excluding patients from rehabilitation.

\section{Conclusions}

Stroke is a neurological condition that often produces disability and requires long hospitalization. Many authors stress the importance of both clinical and organizational conditions in establishing health care policies and in reducing costs. Healthcare systems offer different types of rehabilitative and long-term facilities and data from literature have low validity outside the country in which they were collected. However the organization of our geriatric-rehabilitative area and the multi-professional team implementing an early multi-dimensional assessment may explain the shorter LOS in stroke unit of our patients disposed to intermediate care when compared to other international experiences. Early and significant predictors supporting the choice among intermediate care settings remain uncertain. Until absolute selection criteria for admission to rehabilitation are available, each stroke unit should monitor the process of discharge. In our analysis dysphagia, severe neurological impairment and post-stroke disability were associated with discharge to intermediate care. Younger age and better pre-stroke functional status were associated with discharge to rehabilitation, and older age and hemorrhages to long-term wards. That does not contrast with literature but further studies are warranted to understand if a larger availability of rehabilitative beds may represent a better option for patients presently discharged to a skilled long-term ward and excluded from formal rehabilitation.

\section{References}

1. American Heart Association. Heart disease and stroke statistic - 2004 update. Dallas: American Heart Association; 2003.

2. Govan L, Weir CJ, Langhorne P, for Stroke Unit Trialists' Collaboration. Organised inpatient (stroke unit) care for stroke. Stroke 2008;39:2402-3.

3. Evans A, Perez I, Harraf H, et al. Can differences in management processes explain different outcomes between stroke unit and stroke team care?. Lancet 2001; 358:86-92.
4. Schlegel D, Kolb SJ, Luciano JM, et al. Utility of the NIH Stroke Scale as a predictor of hospital disposition. Stroke 2003;34:134-7.

5. Treger I, Ring H, Schwartz R, et al. Hospital disposition after stroke in a National survey of acute cerebrovascular disease in Israel. Arch Phys Med Rehabil 2008;89: 435-40.

6. Duncan PW, Zorowitz R, Bates B, et al. Management of adult stroke rehabilitation care: a clinical practice guideline. Stroke 2005;36:e100-43.

7. Schelgel DJ, Tanne D, Demchuk AM, et al. Prediction of hospital disposition after thrombolysis for acute ischemic stroke using the National Institutes of Health Stroke Scale. Arch Neurol 2004;61:1061-4.

8. Rundek T, Mast H, Hartmann A, et al. Predictors of resource use after acute hospitalization. The Northern Manhattan Stroke Study. Neurology 2005;55:1180-7.

9. Lai SM, Alter M, Friday G, et al. Disposition after acute stroke: who is not sent home from hospital?. Neuroepidemiology 1998;17:21-9.

10. Bejot Y, Troisgros O, Gremeuax V, et al. Poststroke disposition and associated factors in a population-based study. The Dijon Stroke Registry. Stroke 2012;43:2071-7.

11. Ween JE, Mernoff ST, Alexander MP. Recovery rates after stroke and their impact on outcome prediction. Neurorehabil Neurol Repair 2000;14:229-35.

12. Ring H, Schwartz J, Elazar B, et al. Criteria for referral of CVA patient to rehabilitation. Scand J Rehabil Med Suppl 1985;12:143-7.

13. Hakkennes SJ, Brock K, Hill D. Selection for inpatient rehabilitation after acute stroke: a systematic review of the literature. Arch Phys Med Rehabil 2011;92:2057-70.

14. Wade DT. Selection criteria for rehabilitation services. Clin Rehabil 2003;17:115-8.

15. Masina M, Malservisi S, Domenichini E, et al. Multiprofessional audit supports clinical governance in projectin and implementing a new stroke care area Ital $\mathrm{J}$ Med 2013;7:56-63.

16. Italian Cooperative Research ICR2. Stroke and rehabilitation: Italian Cooperative Research (ICR2). Eur Med Phys 2003;39:7-17.

17. Muscari A, Puddu GM, Santoro N, Zoli M. A simple scoring system for outcome prediction od ischemic stroke. Acta Neurol Scand 2011;124:334-42.

18. Forti P, Maioli F, Procaccianti G, et al. Indipendent predictors od ischemic stroke in the elderly. Prospective data from a stroke unit. Neurology 2013;80:29-38.

19. Hakkennes S, Hill K, Brock K, et al. Accessing inpatients rehabilitation after acute severe stroke: age, mobility, pre-stroke function and hospital unit are associated with discharge to inpatient rehabilitation. Int J Rehabil Res 2012;35:323-9.

20. Maeshima S, Osawa A, Miyazaki Y, et al. Influence of dysphagia on short-term outcome in patients with acute stroke. Am J Phys Med Rehabil 2011;90:316-20.

21. Fink JN, Selim MH, Kumar S, et al. Is the association of National Institutes of Health Stroke Scale scores and acute magnetic resonance imaging stroke volume equal for patients with right- and left-hemisphere ischemic stroke? Stroke 2002;33:954-8.

22. Nakajima M, Kimura K, Ogata T, et al. Relationships between angiographic findings and National Institutes of Health stroke scale score in cases of hyperacute 
carotid ischemic stroke. AJNR Am J Neuroradiol 2004;25:238-41.

23. Saposnik G, Black SE, Hakim A, et al. Age disparities in stroke quality of care and delivery of health services. Stroke 2009;40:3328-35.

24. Lieberman D, Lieberman D. Rehabilitation following stroke in patients aged 85 and above. J Rehabil Res Dev 2005;42:47-53.
25. Bagg S, Pombo AP, Hopman W. Effect of age on functional outcomes after stroke rehabilitation. Stroke 2002;33:179-85.

26. Saposnik G, Kapral MK, Cote R, et al. Is pre-existing dementia an independent predictor of outcome after stroke? A propensity score-matched analysis. J Neurol 2012;259:2366-75. 